\title{
Human scale high-energy physics
}

\author{
Apart from the occasional look backwards, it is almost forgotten that the foundations of high-energy \\ physics were laid not by the design of experiments but by the observation of chance phenomena.
}

Nostalgically, the University of Bristol was last week celebrating the fortieth anniversary of two developments in what is now called high-energy physics which, in retrospect, mark the watershed between the heroic past and the machinebased present. An understanding of how matter is constructed has been an obvious prize since Aristotle's time. Until 1947, progress resulted from people working in ordinary laboratories gleaning what they could, by ingenious design of equipment and sheer hard work, from the chance occurrence of cosmic-ray events.

But already, in 1947, it was clear that the future would lie with the accelerators then being built. That human-scale techniques should have produced in a single year the surprises that then emerged, justifies last weeks' celebrations; that those developments should have turned out to be the last important contributions of British experimentalists to the field may also have been in people's minds.

Historically, 1947 was a chaotic year. Much of the British physics community was occupied with the foundation of the Atomic Energy Research Establishment at Harwell, then a muddy disused airfield, to which groups of academics and their students were repeatedly conducted in the hope that they would stay. (Many did.)

Particle physics, by contrast, was simple. There were protons and neutrons (united in the single entity called a nucleon by the concept of isotopic spin), electrons and positrons (related by Dirac's theory of the latter as holes in a sea of electrons of negative energy), neutrinos (postulated on theoretical grounds by Pauli and Fermi, but not observed) and 'mesotrons' - the particles postulated in 1935 by Yukawa as the mediators of the forces between nucleons, recognized (by their mass, intermediate between that of an electron and proton) among the particles of the cosmic rays and named by C.D. Anderson and S.H. Neddermeyer (Nature 142, 878; 1938) on the basis of observations at the California Institute of Technology. This sparse list of 'fundamental' particles survived the Second World War unchanged.

On reflection, it is odd that mesotrons had been as readily welcomed as Yukawa's hypothetical particles. True, the mass was, as expected, intermediate between that of the electron and the proton (although the difficulty of producing exact estimates suggested to some that mesotrons might not be unique). They were most easily recognized in the component of the cosmic-ray stream penetrating substantial amounts of matter, behaving as if they were 'heavy electrons' (their familiar name) rather than as the mediators of nuclear forces (which should interact strongly with nuclei).

The first development of 1947 was C.F. Powell's success at Bristol in making sense of mesotrons (Lattes, C.M.G., Muirhead, H., Occhialini, G.P.S. and Powell, C.F., Nature 159, 694; 1947). Powell's contribution had been to work away, for the best part of a decade, at the use of photographic emulsions for extracting usable information about cosmic-ray particles from the tracks of exposed silver grains scattered along the paths of quicklymoving charged particles. As the world knows, it was first necessary to persuade manufacturers to make emulsions thick enough to be useful, then to develop the techniques for observing tracks a few $\mu \mathrm{m}$ long and then to work out the rules by which the momentum of a particle could be inferred from the density of exposed grains along a track and from the angles through which it appeared microscopically to have been scattered. The upshot was the great surprise that there are, indeed, two kinds of 'mesotrons' muons and pions, the "heavy electrons" and the Yukawa particles respectively. The masses, as now measured, are the equivalent of 106 and $140 \mathrm{MeV}$ respectively, different enough to account for the indecisions of the 1930s.

The menagerie of fundamental particles was quickly extended with the publication, later that year, of another unexpected cosmic-ray observation - two oddly shaped tracks in separate cosmic-ray cloud chamber photographs taken at the University of Manchester by G.D. Rochester and C.C.Butler (Nature 160, $855 ; 1947)$. One of the two photographs was of a pair of tracks, pointing downwards beneath a lead plate in the chamber, in the V-shaped configuration. The second, even more surprising, shows a particle with minimum ionization suddenly bent through an angle of 19 degrees - and then traversing several centimetres of lead while deviating by only 3 degrees from its original direction.

The two particles were named $\mathrm{V}$ and $\mathrm{K}$ particles. P.M.S. Blackett, the head of the Manchester physics department, energetically arranged that anybody who could operate a cloud chamber in a magnetic field should be sent off, with the appropriate equipment, to look for further examples of the same phenomena, for which the name "strange" was coined because of their surprisingly long lifetime against spontaneous decay. The Pic du Midi, France and the Jungfrau Joch, Switzerland were usual destinations.

There was a famous occasion when Blackett, then also the chairman of the stripling National Research Development Corporation, invited his august committee members to join him on the night train from London to Manchester to inspect the equipment with which this great discovery had been made. Blackett, still very much the naval officer, is believed to have uttered words more appropriate to the forecastle than to the quarter-deck when he found that hapless Rochester had dismantled his cloud chamber for cleaning.

What the cloud-chambers had found was the first evidence of the quantum number now fittingly called strangeness, an attribute of a quark (the notion of which had not then been invented). The cosmic-ray people were cock-a-hoop that so much had been found so quickly. Must not these testimonials to their ingenious craft keep the accelerators at bay?

That was hoping against hope. The accelerators had logic on their side. People began drifting off into other fields. Manchester's head technician, one Thomas Ball, a man with green fingers for building cloud chambers, became the head technician at CERN at Geneva and spent the rest of his working life negotiating contracts with the Brown Bouverie's of this world. It is not that cosmic-ray physics died, but that it became part of observational astrophysics (while the already elaborate counter-controlled cloud chambers became the precursors of the detectors that now dance attendance on the accelerators).

Even so, some may note, it needed the passage of a full quarter of a century before the next quark attribute (charm) was identified by accelerators at Brookhaven and Stanford. What was lost was the fun of seeing how people's ingenuity and dexterity can be made to breed discovery. It is wry that all this was apparent at the point at which cosmic-ray physics had yielded its most spectacular results, which is one reason why Bristol's celebration last week must also have been something of a wake.

John Maddox 